\title{
The use of 3D printing technology in human defect reconstruction-a review of cases study
}

\author{
Sekou Singare*, Chen Shenggui and Li Sheng \\ School of Mechanical Engineering, Dongguan University of Technology, China
}

\begin{abstract}
In this paper, we report the benefit of Computer Tomography $(\mathrm{CT})$, reverse engineering (RE) and rapid prototyping (RP) in individual implant production for maxillofacial surgery. The design and fabrication processes includes: a) processing medical image data of a patient's pathologically defective area having a symmetrical part to construct a three-dimensional (3D) digital model; b) forming an image on the defective side, in this step different techniques are applied for individual cases, including mirroring the non-defect side, implant design from other skull CT data; c) implant geometry modeling; d) 3D printing of the skull and implant model; e) surgical planning and prosthesis fitting; f) casting of a custom-fit titanium implant. Results show that the CAD method can reconstruct the defect after surgical resection. The $3 \mathrm{D}$ printing technique provides an accurate tool for preoperative, surgical simulation. The design and manufacturing time of the bone implant has thus been significantly reduced using the CAD method.
\end{abstract}

\section{Introduction}

The 3D printing has offered aid in medical reconstruction procedures in order to help surgeons reconstruct physical faults and anomalies of their patients. Using a combination of Computer Assisted Design (CAD), high medical skills and 3D printing technologies, it is possible to help patients with craniofacial deformities as birth defects, orthognathic deformities, deformities after malignancy treatment or the consequences of craniofacial injuries of variable severities, resulting in both aesthetic and functional alterations.

Combined with traditional CT scanning techniques 3D printing can be used as instruments for better (three-dimensional) visualization, simulation of procedures and treatment of patients. A customized medical implant and surgical resection template that matches skeletal anatomy can now be accurately designed using the CAD technique. The physical model of the individual implant, template, or skull replica can be produced through $3 \mathrm{D}$ printing, rapid tooling (RT), and computer aided manufacturing/computer numerical control (CAM/ $\mathrm{CNC}$ ) processes [1-15].

This paper presents some clinical cases, where virtual models have been used for surgical preparations and $3 \mathrm{D}$ printing models for manufacturing of implants.

\section{Clinical application}

\section{Case 1}

On March, 2002 a 40 year old women patient nose defect was admitted to a maxillofacial department for defect reconstruction. Data acquisition for 3D modeling was performed with aid of a helical computed tomography (CT) imaging. The 2D CT data were transferred into the MIMICS software to segment CT data so as to expose the anatomy of interest. In this case, the soft tissue is the area of interest in a scan, a threshold value of about 400 is chosen. Then the $3 \mathrm{D}$ volumetric image of the skull was generated for diagnostic and prosthesis designing steps.
Because her nose was completely damaged, a healthy nose data was used to construct the defect part shown in Figure 1a. We choose three points on the standard nose model. The corresponding points are also set on the patient facial model. The healthy nose model is manually fitted their position onto the patient facial image shown in Figure 1b. Using reverse engineering software, the two noses are merged together to form a unique surface. After merging the two noses, the unused parts were cut, then thee solid model is created as shown in Figure 1c, and the design is carefully checked by both designers and surgeons before it is prototyped and produced. Then, the STL file of the solid model is generated and exported to RP machine to build the prosthesis Figure 1d. The SLA model is then used to make the mold (Figure 1e) for manufacturing the silicone rubber prosthesis. Finally, the silicone rubber surface color was adjusted to match the pigmentation of the individual. The retention is made from a medical adhesive which will apply to the back of the prosthesis to hold it in place.

\section{Case 2}

A 50-year-oldman with a large mandible defect underwent initial mandible reconstruction with an intraoperative modeled reconstruction plate (Figure 2a). Few months later after the initial reconstruction, a plate fracture occurred, leading to a nonunion. With the use of manually shaped reconstruction plate, the restoration of facial contour, jaw relationship and condylar position is difficult to attain. These traditional reconstruction plates are difficult to adapt to the anatomical form of the mandible. Failure always occurs at the plates bending area and usually leads to unsatisfactory functional results.

Correspondence to: Sekou Singare, School of Mechanical Engineering, Dongguan University of Technology, Dongguan, Dongguan 523808, China, Tel: 13538661940, E-mail: sekou2d@yahoo.com/sekousingare@hotmail.

Key words: individual template, designed prosthesis, computer-aided reconstruction, $3 D$ printing, rapid tooling

Received: April 04, 2017; Accepted: April 24, 2017; Published: April 27, 2017 


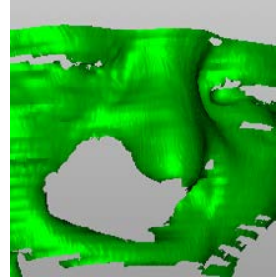

a) Defect nose

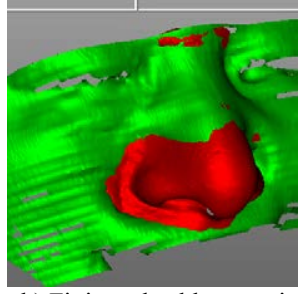

b) Fitting a healthy nose into defect

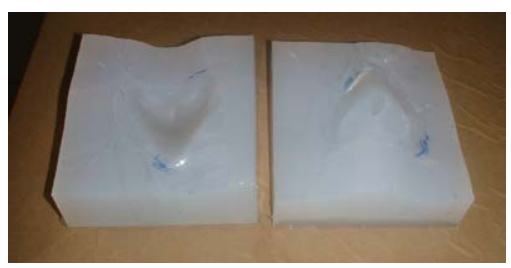

e) Silicone mold

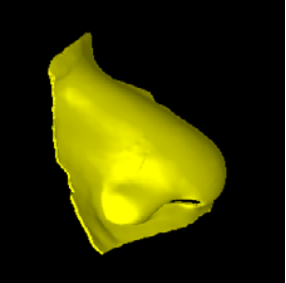

c) CAD model

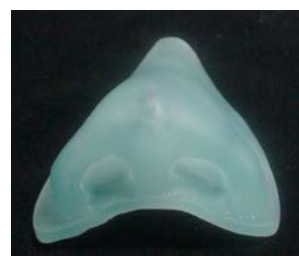

d) $3 \mathrm{D}$ printing model
Figure 1. Reconstruction process of the defect nose.

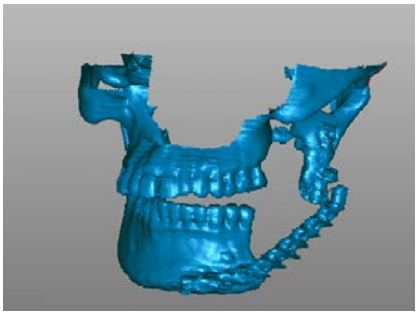
the plate failure

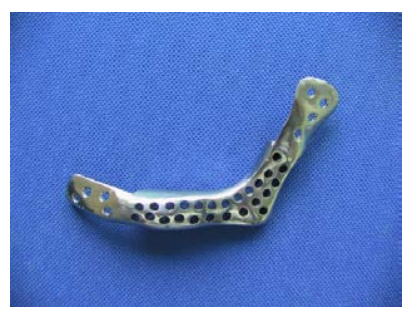

c) Customized titanium prosthesis a) 3D image of the patient skull showing

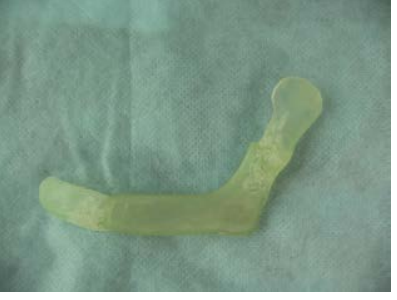

b) SLA prosthesis model

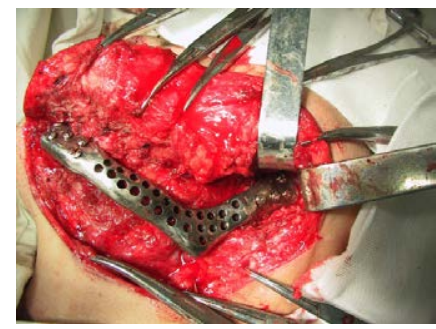

d) Placing the prosthesis during the surgery
Figure 2. Customized titanium implant modeling.

A treatment plan is undertaken to establish the type and purpose of the prosthesis to meet the patient's needs. The treatment plan involves examination of the defect area, and a second surgical operation of the mandible consists of a customized design of the prosthesis had been decide in order to restore bony continuity, facial contour and the mandible function. After CT data acquisition, the CT data were reconstructed and transferred to reverse engineering environment for prosthesis design. We would like to have the mandible without the initial reconstruction plate. The $\mathrm{CT}$ scan of the initial reconstruction plate data needs to be removed from the 3D model of the mandible, the plate is localized from the overall $3 \mathrm{D}$ image, and then the plate image was removed from the overall skull point cloud data. The corresponding right half of the mandible was used to design the prosthesis geometry based on the mirror imaging technique. The mirror plane is created (reference system), and the mirror image is generated by reflecting the data for the non-defect side into the defect side along the mirroring axis. Then the mirror-image template is used to generate the prosthesis pattern (Figure 2). The prosthesis SLA model fabricated by stereolithography machine was used to cast the titanium implant. The prosthesis was then sent to maxillofacial department for surgical operation as shown in Figure 2d. The individual reconstruction implant was inserted. The operation went without complications, the implant profile created good visual appearance of the face. Until nine years of follow-up period, they were no complications or tumor recurrence and the patient was satisfied with the result.

\section{Case 3}

A 43 year-old woman was diagnosed with Benign mandibular tumor, the patient underwent autogenous iliac grafting after segmental mandibular resection. Later the postoperative diagnosis was mandibular osteosarcoma. A radiation therapy was combined with chemotherapy during the postoperative treatment after the tumor recurrence. 3 years later the patient underwent a second surgery, such as extended surgical resection, reconstruction with mandibular titanium plate and pectoralis major myocutaneous pedicled flap, followed by postoperative systemic chemotherapy and immune therapy. Until to day the patient is free of tumor because due to large tissue resection during the second operation, but the titanium reconstruction plate was unable to support the mandibular morphology, as result a complete loss of oral function occurred by the using the traditional reconstruction plate.

A surgical plan of the mandible consists of a customized design of the implant to restore the mandibular continuity, facial contour and the oral function. After CT data acquisition, Mimics software was used to read in the CT slice data from the recent scan, segment the patient's skull detail and generate a three-dimensional computer model of the anatomy, which is imported into reverse engineering software "Geomagic studio version 8" for implant design.

Unlike to case 2, virtual mirroring as a design tool was not feasible due to destruction of the largest part of the mandible. The implant CAD design was based on combination of the mirror imaging technique and another mandible use as template as the one shows in Figure 3.

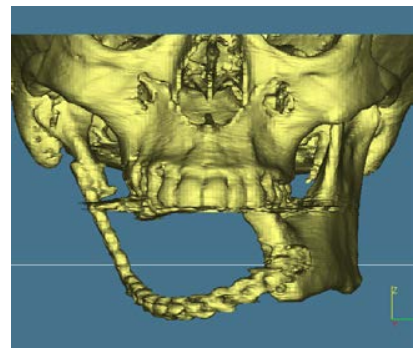

a) Three dimensional model of the patient skull

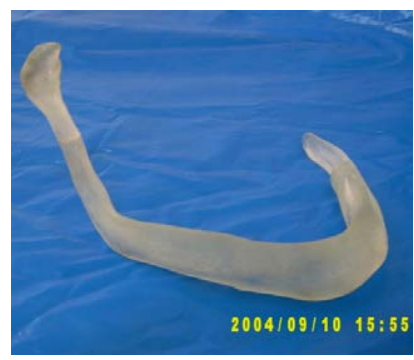

c) $3 \mathrm{D}$ printing model

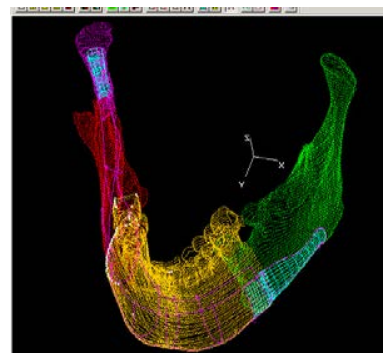

b) Implant design in reverse engineering environment

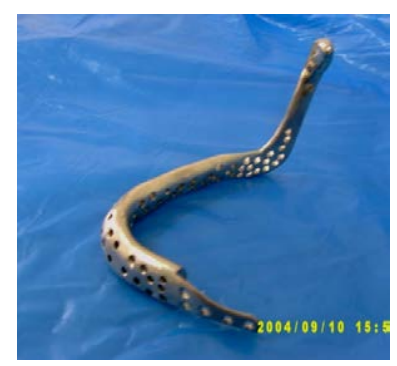

d) Titanium implant model
Figure 3. Customized titanium implant modeling. 
First, the remaining undamaged mandible was mirrored to the defect side. Because the extend of the defect exceed the midline of the chin area, mirroring the undamaged mandible cannot alone produce the overall contour of the implant, so other mandible image was scaled and used as template to complete the implant contour design. The implant SLA model fabricated by stereolithography machine was used to cast the titanium implant (Figure 3c). The implant was then sent to maxillofacial department for surgical operation. There were any difficulties during reconstruction because the individual titanium implant is prefabricated with a geometry fitting to that of the template. As consequence, the implant closes the bone defect perfectly so that the contour is reconstructed precisely. No complications or tumor recurrence were seen in ten year of follow-up period and the patient was satisfied with the result.

\section{Case 4}

As shown in Figure 4a, 34-year-old female patient presented at the hospital reporting pain in her right upper teeth for the past 2 months and nasal blood secretions for the past 20 days. Clinical examination revealed swelling of her right maxilla sinus with middling density and uneven quality, extending into the middle nasal meatus. The inner wall of the maxillary sinus had broken into the nasal septum. Adenocarcinoma of the maxilla was diagnosed.

The patient underwent maxillary bone resection for the first surgery; her wound healed well with no recurrence of the tumor, but with $1 / 3$ facial deformation and severe diplopia. She returned to the hospital for deformation correction and repair.

After importing the $3 \mathrm{D}$ data into the reverse engineering platform a virtual model was built by using mirroring tools. The unaffected right side was mirrored to the affected site. Then the mirror-image template is used to generate the prosthesis pattern. The customized titanium maxillary prosthesis was fabricated and implanted successfully at the correct position during surgery (Figure $4 \mathrm{c}$ ).

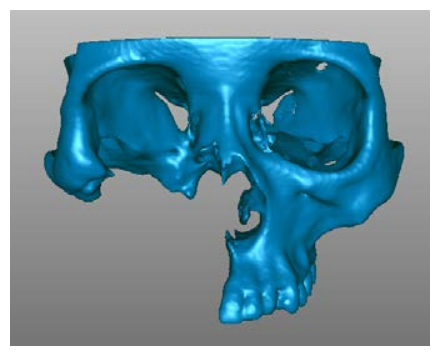

a) 3D image of the patient skull showing the plate failure

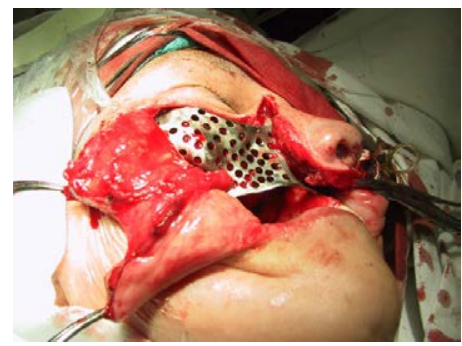

c) Maxilla reconstruction surgery

Figure 4. Customized titanium implant modeling for maxilla reconstruction.

\section{Case 5}

The modeling of a mandibular bone loss was chosen as a case study to show the possibility of using technology of rapid prototyping and rapid machining in medical implant producing. After a thorough clinical assessment, a computed tomography (CT) scan was performed using a standard craniofacial protocol. The CT raw data is loaded into medical imaging software (Mimics 8.1) which is processed to generate a three-dimensional image of the patient's bone structure (Figure 5-1).

A clinical assessment of the 3D image was performed to evaluate the location of the mandible segment. The $3 \mathrm{D}$ image showed that the mandible had shifted from it normal position. Therefore to design the implant with respect to the correct position of the mandible, the deformed mandible should be accurately repositioned to its correct anatomical position, so as to optimize the surgical procedures and to improve the surgical outcome. The 3D models provide a detailed overview to allow specialists to comprehend the location, shape and extent of the pathology.

After evaluation of the pathology, the reconstructed 3D voxel image is converted into a point cloud and imported by reverse engineering software (Geomagic Studios (Raindrop Inc)) to design the custom-fit implant.

After the surgical resection of the mandible to remove the tumor area, the jaw is moved out of its normal position. The design of the custom implant in this abnormal position of the jaw can affect the normal movement of the jaw during chewing and speech. Furthermore, such a situation can produce a facial asymmetry. In order to virtually restore the mandible into proper anatomic position, the mandible and skull must be independently separated from the overall 3D image. The mandible is first split from the skull, and then repositioned it to the normal position through rotation (Figure 5-2).

When alignment between the mandible and maxilla are established, a suitable mirroring plan for the mandible is created in the midline plane along the nose-bridge to mirror the non-affected side image (Figure 5-3 (a)). The mirror image of the left is formed based on the axis of symmetry of the 3D digital model (Figure 5-3 (b)). The mirror image is then overlaid on the original image to form a composite image with a non-overlapping area wherein the implant will be fitted. A digital implant is then generated by cutting off the non-overlapping area of the mirror image (Figure 5-3(c)). Next, NURBS patches are used to fit across these geometrical contours to generate the prosthesis CAD surface model, and the surface is thickened to generate the solid model.
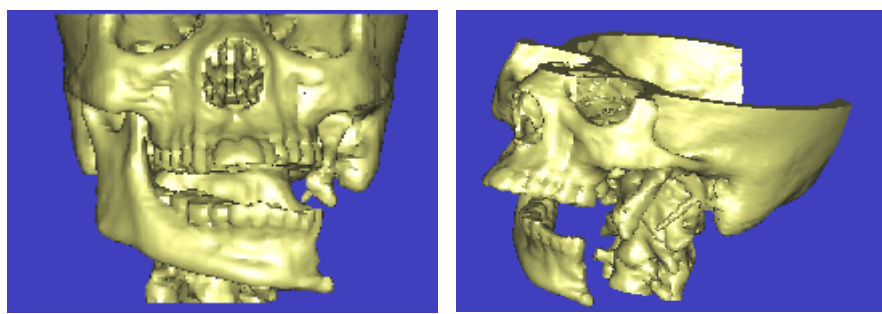

Figure 5-1. 3D virtual surgical planning.
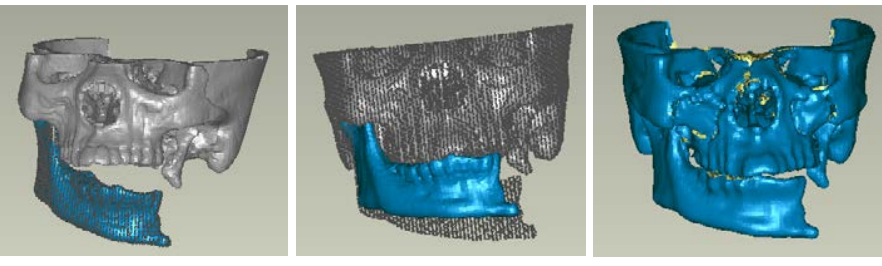

Figure 5-2. Positioning of the mandible into normal position. 
The CAD data of skull and the prosthesis are translated into an STL file format and imported into the RP machine to fabricate the physical object. Finally, the stereolithography apparatus (SLA) prosthesis pattern (Figure 5-4 a) is directly used in investment casting such as Quick-Cast for production of the titanium prosthesis (Figure 5-4 b).

\section{Conclusion}

Treatment of patients after injures or diseases resulting in deformational consequences usually requires the implantation of either autologous tissues or biocompatible / biodegradable implants that replace missing parts of the tissue, usually bone.

In maxillomaxilo-facial treatments, implants have to fulfill an aesthetic function, therefore, the possibilities of their prefabrication by means of serial production are very limited. Design and fabrication of reconstruction implant using $\mathrm{CAD}$ and $3 \mathrm{D}$ printing is reported. The process involves imaging bone structure using CT scans; conversion of two dimensional slices into three dimensional models, implant geometry design in reverse engineering based on $3 \mathrm{D}$ reconstructed model, and creation of physical model of a patient's bone structure and implant using 3D printing technique for implant fit evaluation, planning and simulating surgical procedures, and defect reconstruction . The physical model has been used for visualisation by many surgeons in order to make better judgement on further treatment plans. With regards to the manufacturing of implant, the medical models obtained by $3 \mathrm{D}$ printing are normally used indirectly, as masters, to produce prosthesis in biocompatible conventional materials (e.g. titanium, cobalt-chrome alloys etc.). As result the implant produce by $3 \mathrm{D}$ printing technique can lead to improved surgery results, less blood loss and faster recovery for the patient.

The presented case studies show the great potential of 3D printing technologies in medical applications. Two patients were primarily reconstructed by using commercial plate, reconstruction plate failure occur in both cases, to further improve the oromandibular defect reconstruction after resection, a custom made implant is fabricated using CT data, CAD and 3D printing technique. In limited mandible defects, shape and size of the reconstruction implant could be designed using mirroring technique; good results could be achieved with this simple planning method (case 2). In larger defects (case 3) simple mirroring tools are not feasible. The implant CAD design was based on

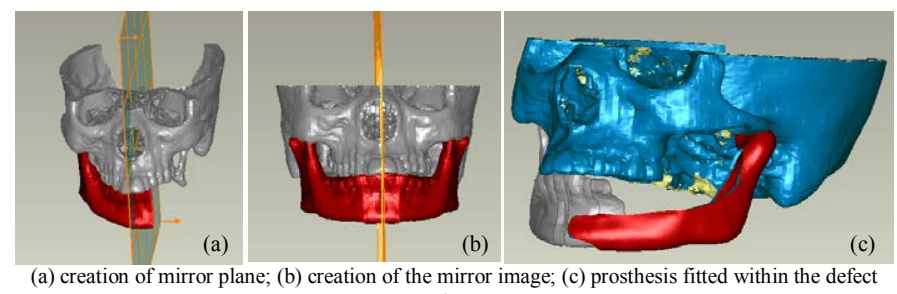

site.

Figure 5-3. Design of custom implant.
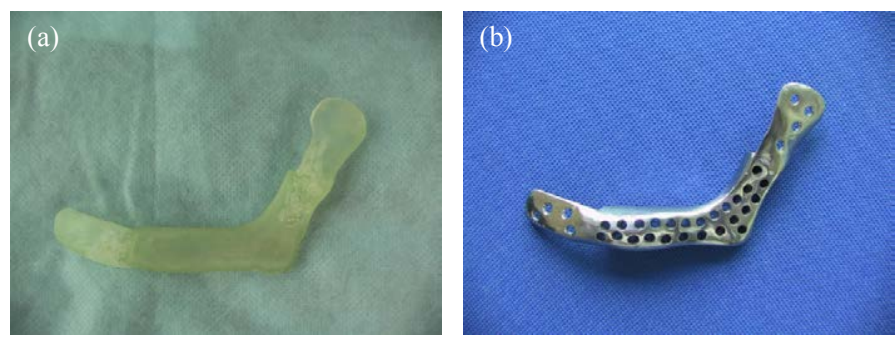

Figure 5-4. (a) SLA implant model; (b) Titanium implant model. combination of the mirror imaging technique and another mandible use as template. There were no complications in all patients who underwent reconstruction using $3 \mathrm{D}$ printing implants in ten year of follow-up, all of these patients regained full oral function. The uses of $\mathrm{CT} / \mathrm{CAD} / 3 \mathrm{D}$ printing technique have facilitated the mandible reconstruction surgery, and offer a better life to the patient's.

\section{Acknowledgements}

This project is supported by National Natural Science Foundation of China (Grant No. 51445008), Science and Technology Planning Project of Guangdong Province (Project No. 2013B090500130, 2015A010101305, 2013B090600047), Project supported by Guangdong Provincial Key Laboratory construction project of China (2011A060901026).

\section{References}

1. Morrison DA, Guy DT, Day RE, Lee GY (2011) Simultaneous repair of two large cranial defects using rapid prototyping and custom computer-designed titanium plates: a case report. Proc Inst Mech Eng H 225: 1108-1112. [Crossref]

2. Lee SC, Wu CT, Lee ST, Chen PJ (2009) Cranioplasty using polymethyl methacrylate prostheses. J Clin Neurosci 16: 56-63. [Crossref]

3. Singare S, Dichen L, Bingheng L, Zhenyu G, Yaxiong L (2005) Customized design and manufacturing of chin implant based on rapid prototyping. Rapid Prototyping Journal 11: 113-118

4. Singare S, Lian Q, Wang WP, Wang J, Liu Y, et al. (2009) Rapid prototyping assisted surgery planning and custom implant design. Rapid Prototyping Journal 15: 19-23.

5. Winder J, Bibb R (2005) Medical rapid prototyping technologies: state of the art and current limitations for application in oral and maxillofacial surgery. J Oral Maxillofac Surg 63: 1006-1015. [Crossref]

6. Müller A, Krishnan KG, Uhl E, Mast G (2003) The application of rapid prototyping techniques in cranial reconstruction and preoperative planning in neurosurgery. $J$ Craniofac Surg 14: 899-914. [Crossref]

7. Eppley BL, Sadove AM (1998) Computer-generated patient models for reconstruction of cranial and facial deformities. J Craniofac Surg 9: 548-556. [Crossref]

8. Zhou LB, Shang HT, He LS, Bo B, Liu GC, et al. (2010) Accurate reconstruction of discontinuous mandible using a reverse engineering/computer-aided design/rapid prototyping technique: a preliminary clinical study. J Oral Maxillofac Surg 68: 2115 2121. [Crossref]

9. Rotaru H, Stan H, Florian IS, Schumacher R, Park YT, et al. (2012) Cranioplasty With Custom-Made Implants: Analyzing the Cases of 10 Patients. J Oral Maxillofac Surg 70: e169-e176. [Crossref]

10. Aakash Arora, Abhay N. Datarkar, et al. (2013) Custom-Made Implant for Maxillofacia Defects Using Rapid Prototype Models. J Oral Maxillofac Surg 71: e104-e110. [Crossref]

11. Wang G, Li J, Khadka A, Hsu Y, Li W, et al. (2012) CAD/CAM and rapid prototyped titanium for reconstruction of ramus defect and condylar fracture caused by mandibular reduction. Oral Surg Oral Med Oral Pathol Oral Radiol 113: 356-361. [Crossref]

12. Mustafa SF, Evans PL, Bocca A, Patton DW, Sugar AW, et al. (2011) Customized titanium reconstruction of post-traumatic orbital wall defects: a review of 22 cases. Int J Oral Maxillofac Surg 40: 1357-1362. [Crossref]

13. Taft RM, Kondor S, Grant GT (2011) Accuracy of rapid prototype models for head and neck reconstruction. J Prosthet Dent 106: 399-408. [Crossref]

14. Markiewicz MR, Bell RB (2011) The Use of 3D Imaging Tools in Facial Plastic Surgery Review Article. Facial Plast Surg Clin North Am 19: 655-682. [Crossref]

15. Lai JB, Sittitavornwong S, Waite PD (2011) Computer-Assisted Designed and Computer-Assisted Manufactured Polyetheretherketone Prosthesis for Complex Fronto-Orbito-Temporal Defect. J Oral Maxillofac Surg 69: 1175-1180. [Crossref]

Copyright: (C2017 Singare S. This is an open-access article distributed under the terms of the Creative Commons Attribution License, which permits unrestricted use, distribution, and reproduction in any medium, provided the original author and source are credited. 